\title{
Gamma discrimination in pillar structured thermal neutron detectors
}

Q. Shao, R. P. Radev, A. M. Conway, L. F. Voss, T. F. Wang, R. J. Nikolic, N. Deo, C. L. Cheung

March 30, 2012

SPIE defense security and sensing 2012 Baltimore, MD, United States April 23, 2012 through April 27, 2012 
This document was prepared as an account of work sponsored by an agency of the United States government. Neither the United States government nor Lawrence Livermore National Security, LLC, nor any of their employees makes any warranty, expressed or implied, or assumes any legal liability or responsibility for the accuracy, completeness, or usefulness of any information, apparatus, product, or process disclosed, or represents that its use would not infringe privately owned rights. Reference herein to any specific commercial product, process, or service by trade name, trademark, manufacturer, or otherwise does not necessarily constitute or imply its endorsement, recommendation, or favoring by the United States government or Lawrence Livermore National Security, LLC. The views and opinions of authors expressed herein do not necessarily state or reflect those of the United States government or Lawrence Livermore National Security, LLC, and shall not be used for advertising or product endorsement purposes. 


\title{
Gamma Discrimination in Pillar Structured Thermal Neutron Detectors
}

\author{
Q. Shao ${ }^{\text {a }}$, R. P. Radev ${ }^{\text {a }}$, A. M. Conway ${ }^{a}$, L. F. Voss ${ }^{a}$, T. F. Wang ${ }^{a}$, and R. J. Nikolic ${ }^{* a}$, \\ N. Deo ${ }^{\mathrm{b}}$ and C. L. Cheung ${ }^{\mathrm{b}}$ \\ ${ }^{a}$ Lawrence Livermore National Laboratory, 7000 East Avenue, Livermore, CA 94550 USA \\ ${ }^{b}$ Department of Chemistry, University of Nebraska-Lincoln, 1400 R Street, Lincoln, NE 68588 USA
}

\begin{abstract}
Solid-state thermal neutron detectors are desired to replace ${ }^{3} \mathrm{He}$ tube based technology for the detection of special nuclear materials. ${ }^{3} \mathrm{He}$ tubes have some issues with stability, sensitivity to microphonics and very recently, a shortage of ${ }^{3} \mathrm{He}$. There are numerous solid-state approaches being investigated that utilize various architectures and material combinations. By using the combination of high-aspect-ratio silicon PIN pillars, which are $2 \mu \mathrm{m}$ wide with a $2 \mu \mathrm{m}$ separation, arranged in a square matrix, and surrounded by ${ }^{10} \mathrm{~B}$, the neutron converter material, a high efficiency thermal neutron detector is possible. Besides intrinsic neutron detection efficiency, neutron to gamma discrimination is an important figure of merit for unambiguous signal identification. In this work, theoretical calculations and experimental measurements are conducted to determine the effect of structure design of pillar structured thermal neutron detectors including: intrinsic layer thickness, pillar height, substrate doping and incident gamma energy on neutron to gamma discrimination.
\end{abstract}

Keywords: neutron detector, gamma discrimination, pillar, boron, Monte Carlo simulation, COMSOL simulation

\section{INTRODUCTION}

Because thermal neutron detectors are sensitive to gamma rays, and most nuclear materials emit 10 or more times as many gamma rays as neutrons, the gamma ray sensitivity of a neutron detector is an important figure-of-merit. Gamma rays can deposit energy to detector by Compton scattering or photoelectric effect and generate electron-hole pairs which create the electronic current indicating the presence of radiation. The current that is measured can be generated by either radiation from gamma or neutron interaction. It is desirable to have registration of only the intended radiation source. In order to effectively shield gamma-rays, thick lead bricks can be piled around the detector due to its high density of $11.35 \mathrm{~g} / \mathrm{cm}^{3}$ and high atomic number of 82 [1]. But lead shielding makes the detection system heavy and less portable. It is important to design and optimize the materials and device structures for high neutron detection efficiency with excellent neutron-to-gamma discrimination.

${ }^{3} \mathrm{He}$ tubes [2] are the mostly widely used thermal neutron detectors due to their high capture cross-section of thermal neutrons (3837 barns), high interaction probability with thermal neutrons and low interaction probability with gamma rays. Neutron-to-gamma separation can be done by inspection of amplitudes of registered pulses [3]. Due to shortage of ${ }^{3} \mathrm{He}$, sensitivity to microphonics, and usability in the field, solid state detectors attract much attention as an alternative technology to replace ${ }^{3} \mathrm{He}$ tubes.

The detector under development is based on high aspect ratio P-I-N diodes filled with ${ }^{10} \mathrm{~B}$, the neutron conversion material [4-9]. Thermal neutrons have a low probability of interacting with conventional semiconductor materials. Thus, a two step detection process is generally required. First, the thermal neutrons are converted to energetic ions by a material with a high thermal neutron cross-section. In our device ${ }^{10} \mathrm{~B}$ is used (its cross-section for thermal neutrons is 3,837 barns) resulting in the following reaction: $\mathrm{n}+{ }^{10} \mathrm{~B} \rightarrow \alpha+{ }^{7} \mathrm{Li}$. Second, these ions are collected using a reverse biased semiconductor diode. Three major criteria drive the optimal design of thermal neutron detectors: sufficient thickness of neutron convertor material $\left(50 \mu \mathrm{m}\right.$ in $\left.{ }^{10} \mathrm{~B}\right)$, high probability of ion energy deposition within semiconductor

*nikolic1@1lnl.gov; phone: 1925 423-7389 
detector (ion track length $3 \mu \mathrm{m}$ in ${ }^{10} \mathrm{~B}$ ), and large discrimination between gamma ray events and thermal neutron events. Using a three-dimensionally integrated approach, very high detection efficiency is possible because the geometrical constraints on the converter material thickness are decoupled from the limitation of the ion track length, as shown in Figure 1. In this case, the ${ }^{10} \mathrm{~B}$ thickness is defined by the pillar height (etch depth) so as to absorb the thermal neutrons. The pillar pitch is defined lithographically to allow the highest possible interaction of the energetic ions with the semiconductor pillars. When the 3D pillar detector is scaled to $50 \mu \mathrm{m}$, high efficiency $(>50 \%)$ and high neutron-togamma discrimination $\left(>10^{5}\right)$ is predicted [4-8]. In this work, the effects of intrinsic layer thickness, pillar height, substrate doping as well as incident gamma energy on gamma discrimination are investigated.

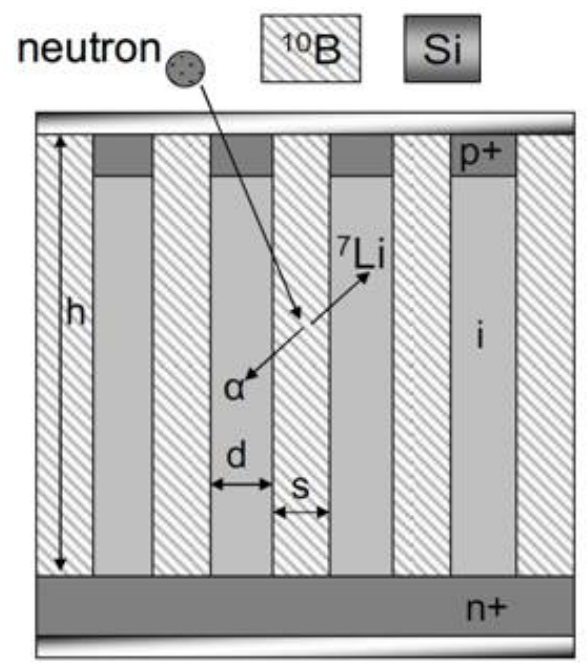

Figure 1 Schematic of a pillar structured thermal neutron detector, h: pillar height $(50 \mu \mathrm{m})$, d: pillar size $(2 \mu \mathrm{m})$, s: pillar separation $(2 \mu \mathrm{m})$

\section{DESIGN CONSIDERATIONS}

Figure 2 shows the schematic of a pillar structured thermal neutron detector. $\mathrm{H}_{1}, \mathrm{H}_{2}$ and $\mathrm{H}_{3}$ denote pillar height, intrinsic layer thickness below pillars and substrate thickness, respectively. There are two basic processes in radiation detection: radiation absorption and generated carrier transport. For neutron detection, the absorption only takes place in neutron conversion material $\left({ }^{10} \mathrm{~B}\right)$ which is defined by the area void of pillars. ${ }^{10} \mathrm{~B}$ should be sufficiently thick for neutron absorption or roughly three times the mean free path $(18 \mu \mathrm{m})$ of neutrons in ${ }^{10} \mathrm{~B}$. Generated carriers are swept into $\mathrm{n}^{+}$and $\mathrm{p}^{+}$layer by electric field induced by either built-in voltage or external reverse bias. For gamma detection, the absorption takes place in the entire silicon portion $\left(\mathrm{p}^{+}\right.$, intrinsic, $\mathrm{n}^{+}$substrate). Carriers generated in intrinsic region $\left(\mathrm{H}_{1}\right.$ and $\left.\mathrm{H}_{2}\right)$ are collected by carrier drift induced by electric field, and carriers generated in $\mathrm{p}^{+}$region and $\mathrm{n}^{+}$substrate $\left(\mathrm{H}_{3}\right)$ are collected by carrier diffusion. The portion of collected carriers in the high doping region depends on carrier diffusion length which is determined by doping concentration.

In general, increasing pillar height $\left(\mathrm{H}_{1}\right)$ enhances both neutron and gamma absorption. Increasing $\mathrm{H}_{2}$ only increases gamma absorption since the intrinsic layer below pillars is only sensitive to gamma-rays and void of ${ }^{10} \mathrm{~B}$ which is required for neutron absorption. This layer should be reduced as much as possible. Increasing $\mathrm{H}_{3}$ enhances gamma absorption, but the gamma counts will saturate when $\mathrm{H}_{3}$ is much thicker than carrier's diffusion length.

The value of neutron-to-gamma discrimination which is defined by ratio of neutron efficiency to gamma efficiency depends on structure geometry and substrate doping concentration. In this section, all of these effects are discussed in detail. 


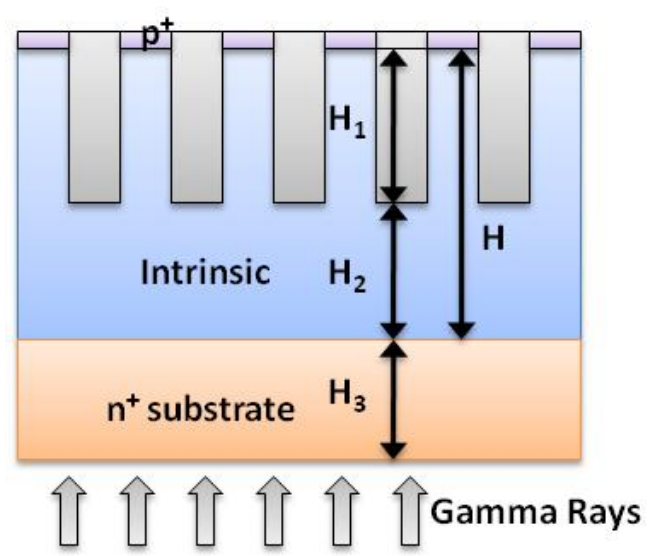

Figure 2 Schematic of a pillar structured thermal neutron detector with gamma radiation

\subsection{Intrinsic layer thickness}

Pillar arrays are formed by deep reactive ion etch on a silicon substrate with PIN structure. The pillars can be etched all the way through intrinsic layer for the best gamma discrimination. The excess intrinsic layer below the pillars makes the detector more sensitive to gamma ray without further improvement of neutron detection efficiency. Figure 3 shows the measured gamma discrimination with gamma energy of $662 \mathrm{keV}$ as a function of total thickness of intrinsic layer. The total thickness $(\mathrm{H})$ is the sum of pillar height $\left(\mathrm{H}_{1}\right)$ and the thickness of intrinsic layer below pillars $\left(\mathrm{H}_{2}\right)$ as shown in Fig. 2. The discrimination value decreases from $1.3 \times 10^{7}$ down to 44 as the intrinsic layer thickness increases to $190 \mu \mathrm{m}$. Sensitivity of ${ }^{3} \mathrm{He}$ tube to gamma-rays is a function of the pressure of ${ }^{3} \mathrm{He}$ gas and tube volume. The sensitivity increases with gas pressure for a fixed volume. Typically ${ }^{3} \mathrm{He}$ tube has gamma discrimination in the range of $10^{5}-10^{9}[3,10]$. In order to obtain gamma discrimination of $10^{5}$, the total intrinsic thickness in pillar detector should be less than $\sim 80 \mu \mathrm{m}$.

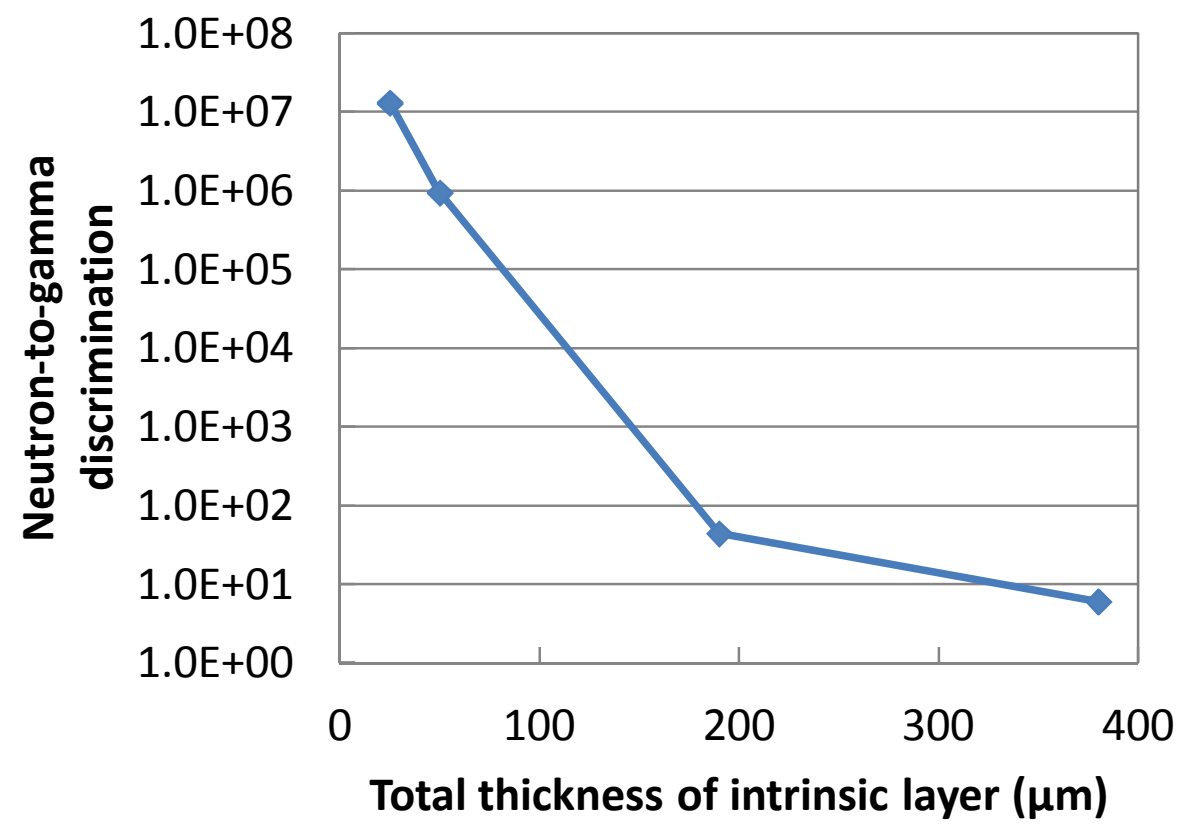

Figure 3 Measured gamma discrimination with gamma energy of $662 \mathrm{keV}$ as a function of total thickness of intrinsic layer (Pillar heights are $25 \mu \mathrm{m}, 50 \mu \mathrm{m}, 50 \mu \mathrm{m}$ and $50 \mu \mathrm{m}$ for four samples with $25 \mu \mathrm{m}, 50 \mu \mathrm{m}, 190 \mu \mathrm{m}$ and $380 \mu \mathrm{m}$ intrinsic layers respectively) 


\subsection{Pillar height}

Gamma efficiency and neutron-to-gamma discrimination as a function of pillar height are simulated by MCNP [11] (Figure 4). Gamma energies in the simulation are $0.66 \mathrm{MeV}, 1 \mathrm{MeV}$ and $1.3 \mathrm{MeV}$. The results show that gamma efficiency linearly increases with pillar height of interest $(25 \mu \mathrm{m}$ to $75 \mu \mathrm{m})$ for all the gamma energies considered. As the gamma energy decreases the gamma efficiency increases due to the higher probability of the gamma interaction with the Si pillar array. The discrimination value increases with pillar height up to $50 \mu \mathrm{m}$, after which the neutron efficiency starts to saturate. Note that greater than $90 \%$ of neutrons normally incident on the surface are absorbed by $50-\mu \mathrm{m}$-thick ${ }^{10} \mathrm{~B}$. Beyond the saturation point $(50 \mu \mathrm{m}$ pillar height), the discrimination value decreases with pillar height, because the increasing of neutron efficiency becomes slower than that of gamma efficiency. The thermal neutron detection efficiencies with varied pillar heights are obtained from Ref. [7]. In general, the discrimination is in the range of $2 \times 10^{5}$ to $8 \times 10^{5}$ for gamma energies of interest. The optimized pillar height is $50 \mu \mathrm{m}$ in terms of thermal neutron detection efficiency and gamma discrimination. The low gamma discrimination value is due to the low linear attenuation coefficient $(\mu)$, which is corresponding to the interaction probability per unit distance. The low $\mu$ is responsible for the small gamma counts being registered and correspondingly large discrimination value.

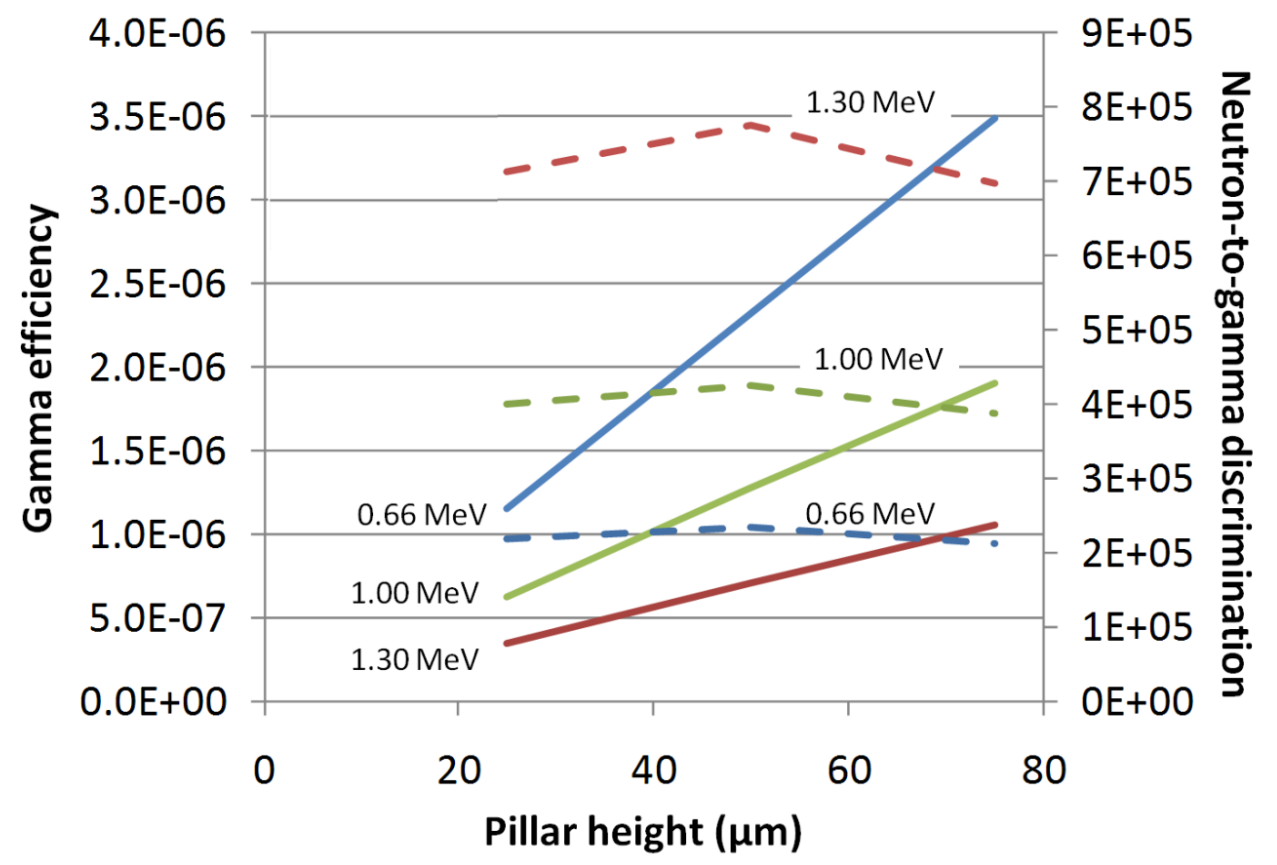

Figure 4 Simulated gamma efficiency (solid lines) and neutron-to-gamma discriminations (dotted lines) for a pillar diameter of 2 $\mu \mathrm{m}$ and pillar separation of $2 \mu \mathrm{m}$ versus pillar height (LLD setting: $50 \mathrm{keV}$ )

\subsection{Substrate doping and thickness}

In addition to intrinsic layer absorption, gamma-rays can also be absorbed in the substrate with the same absorption rate. The charge carriers generated by the radiation in a highly doped substrate recombine quickly due to their short lifetimes. But minority carriers still have the possibility to contribute to the output signal by diffusing into the intrinsic layer and finally collected by electrodes. Table 1 lists the electrical properties of minority carrier (hole) in a highly doped $\mathrm{n}$ type $\mathrm{Si}$ substrate. The high flux gamma radiation on pillar detectors is simulated by COMSOL MULTIPHYSICS [12]. In the

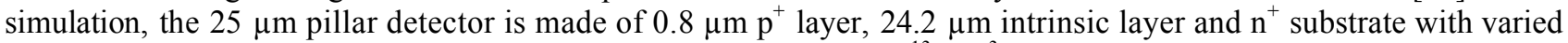
thickness. The device is biased to fully deplete lightly doped $\left(3 \times 10^{13} \mathrm{~cm}^{-3}\right)$ intrinsic layer. High flux gamma rays are normally impinging the device from the $\mathrm{n}^{+}$substrate. The flux of gamma rays can be expressed by $I_{x}=I_{0} \exp (-\alpha x)$, where $\alpha$ is linear attenuation coefficient, $I_{0}$ is incident flux and $x$ is the travel distance. The linear attenuation coefficients are $0.68 \mathrm{~cm}^{-1}, 0.18 \mathrm{~cm}^{-1}$, and $0.14 \mathrm{~cm}^{-1}$ for gamma energies of $60 \mathrm{keV}, 662 \mathrm{keV}$ and $1.3 \mathrm{MeV}$ respectively. The particular energies are chosen because they are signature gamma energies of gamma sources: ${ }^{241} \mathrm{Am},{ }^{137} \mathrm{Cs},{ }^{60} \mathrm{Co}$ respectively. The 
charge carrier generation rate in a pillar is describe by $G_{x}=\alpha I_{x} E_{\gamma} / E_{i o n}$, where $E_{\gamma}$ is the energy deposited onto pillars and $E_{\text {ion }}$ is the ionization energy of electron hold pairs $(\sim 3.62 \mathrm{eV})$ in silicon. Gamma counts are proportional to the generation rate. As shown in Figure 5, the tendency of substrate thickness dependence remains the same regardless of gamma energies. The gamma counts saturate ( $95 \%$ of maximum gamma counts) at $\sim 23 \mu \mathrm{m}$ due to the minority carrier (hole) diffusion length. Figure 6 shows the effect of substrate doping on gamma counts with incident gamma energy of 1.3 MeV. The gamma counts are the same for both doping concentrations when substrates are thin enough to allow all generated minority carriers diffusion into intrinsic region. As compared to $1 \mu \mathrm{m}$ substrate thickness $30 \%$ more gamma counts are expected for substrate thickness above $40 \mu \mathrm{m}$ for doping of $1 \times 10^{18} \mathrm{~cm}^{-3}$. But, only $5 \%$ more gamma counts are expected for substrate thickness above $5 \mu \mathrm{m}$ for doping of $1 \times 10^{19} \mathrm{~cm}^{-3}$. Therefore, highly doped substrate is required to make less gamma detection.

Table 1 Properties of minority carrier (hole) in highly doped n-Si substrate

\begin{tabular}{|c|c|c|c|}
\hline Doping concentration $\left(\mathrm{cm}^{-3}\right)$ & Mobility $\left(\mathrm{cm}^{2} / \mathrm{Vs}\right)$ & Lifetime $(\mu \mathrm{s})$ & Diffusion Length $(\mu \mathrm{m})$ \\
\hline $1 \times 10^{18}$ & 89.2 & 0.248 & 7.57 \\
\hline $1 \times 10^{19}$ & 50.7 & 0.025 & 1.81 \\
\hline
\end{tabular}

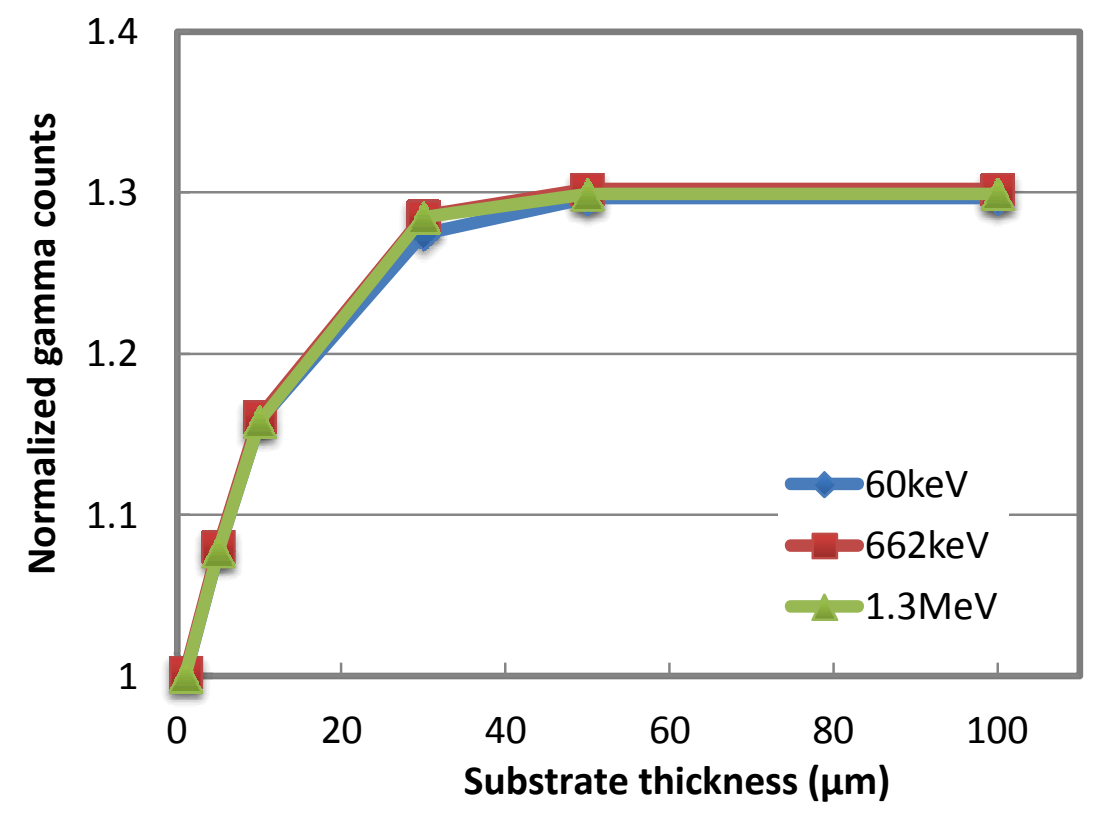

Figure 5 Gamma counts as a function of substrate thickness (doping concentration of $1 \times 10^{18} \mathrm{~cm}^{-3}$ ) normalized to that of $1 \mu \mathrm{m}$ thick substrate for varied gamma energies 


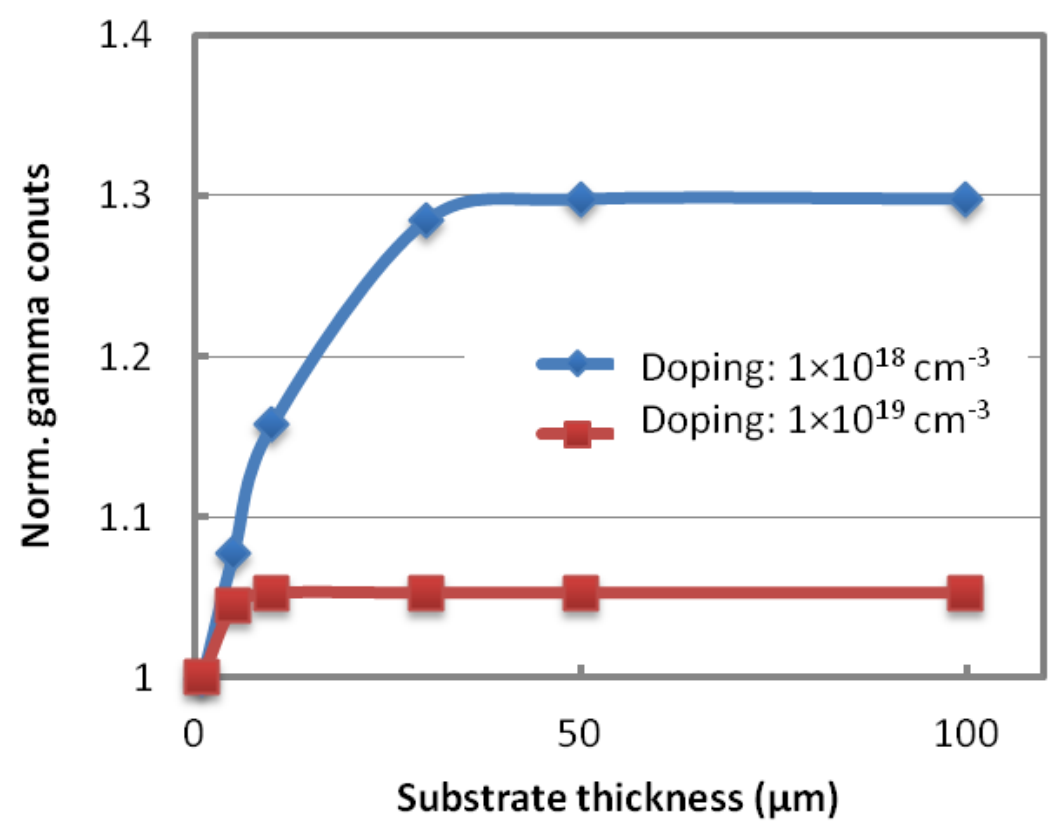

Figure 6 Gamma counts as a function of substrate thickness normalized to that of $1 \mu \mathrm{m}$ thick substrate for varied substrate doping concentration $\left(1 \times 10^{18} \mathrm{~cm}^{-3}\right.$ and $\left.1 \times 10^{19} \mathrm{~cm}^{-3}\right)$

\section{EXPERIMENTAL}

Three devices based on the P-I-N structure $\left(0.8 \mu \mathrm{m} \mathrm{p}^{+}\right.$layer, $24.2 \mu \mathrm{m}$ intrinsic layer and $\mathrm{n}^{+}$substrate $)$were investigated in this experiment. The pillar heights for these devices are $0,12 \mu \mathrm{m}$ and $25 \mu \mathrm{m}$ respectively. The pillar arrays are filled with ${ }^{10} \mathrm{~B}$ by low pressure chemical vapor deposition [13]. The physical sizes of devices are $2 \times 2 \mathrm{~mm}^{2}$. All devices were operated at $0 \mathrm{~V}$ and irradiated with ${ }^{137} \mathrm{Cs}$ gamma sources with activity of $5.64 \times 10^{5} \mathrm{MBq}$ in Radiation Calibration Lab at Lawrence Livermore National Laboratory. Figure 7 shows the schematic of gamma radiation setup. The devices were placed $30 \mathrm{~cm}$ away from gamma sources. The irradiation time was set to 3600 seconds. The background noise spectrums without radiation exposure were also measured for 3600 seconds. A standard Ortec 142 preamplifer and Ortec 572 amplifier together with AMPTEK 8000A multichannel analyzer were used for data collection.

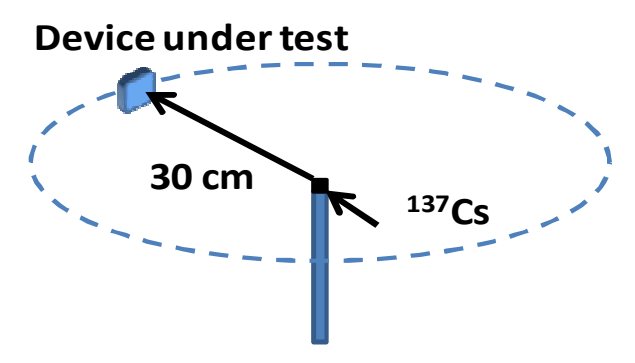

Figure 7 Schematic of gamma irradiation setup

\section{RESULTS AND DISCUSSION}

The gamma pulse height spectra after subtraction of background noise for three $24.2 \mu \mathrm{m}$ intrinsic layer devices are shown in Figure 8. The detected gamma counts are shown in logarithmic scale as a function of energy. The maximum energies detected by flat diode, $12 \mu \mathrm{m}$ pillar detector and $25 \mu \mathrm{m}$ pillar detector are approximately 500,300 and $100 \mathrm{keV}$, respectively. The flat diode is most sensitive to gamma rays because of its largest portion of silicon. Gamma efficiency is 
extracted by the ratio of gamma counts with energy above low level discriminator (LLD) to incident number of gamma rays. The gamma efficiencies as a function of LLD are shown in Figure 9. For a given LLD setting, the flat diode has the highest gamma efficiency and $25 \mu \mathrm{m}$ pillar detector has the lowest gamma efficiency. At $50 \mathrm{keV}$, gamma efficiency of $25 \mu \mathrm{m}$ pillar detector is $4 \times 10^{-7}$. The simulated gamma efficiency with the same structure and gamma ray energy of 660 $\mathrm{keV}$ is $1.2 \times 10^{-6}$ as shown in Figure 3. The low measured efficiency is probably due to the poor charge carrier collection resulting from charge recombination at rough pillar sidewalls.

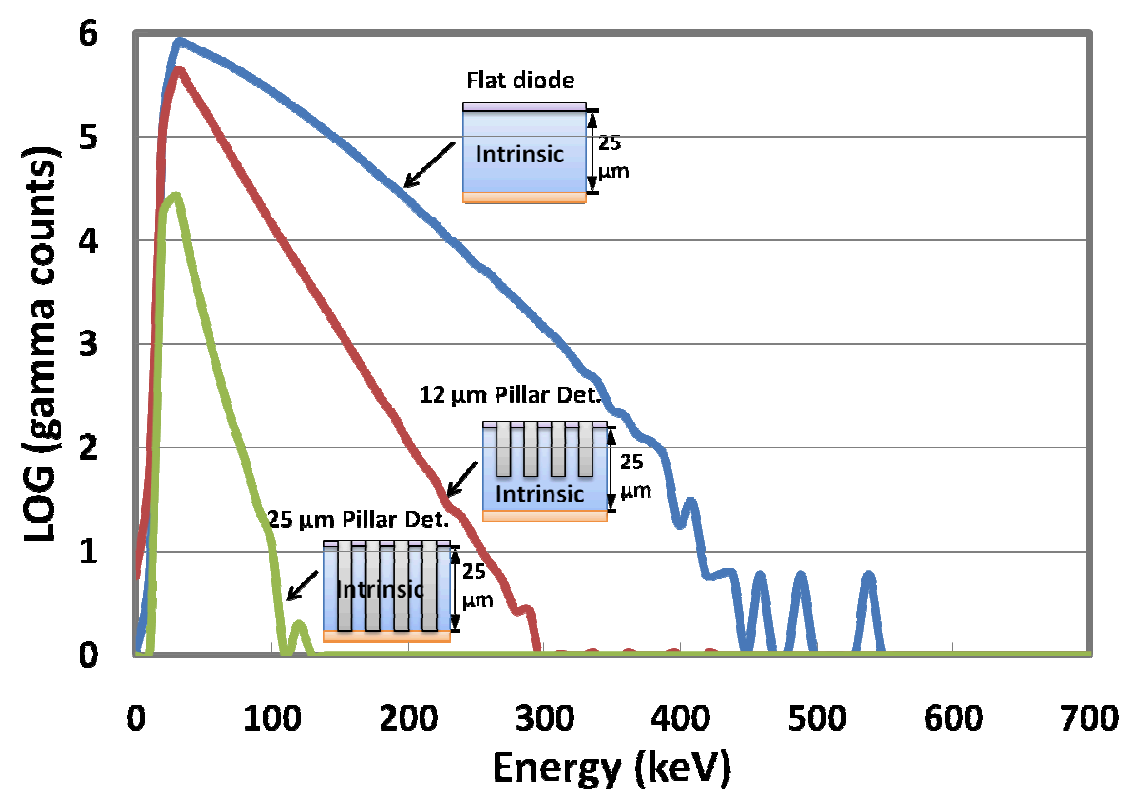

Figure 8 Gamma pulse height spectra of three devices with $25 \mu \mathrm{m}$ intrinsic layer after subtraction of background noise

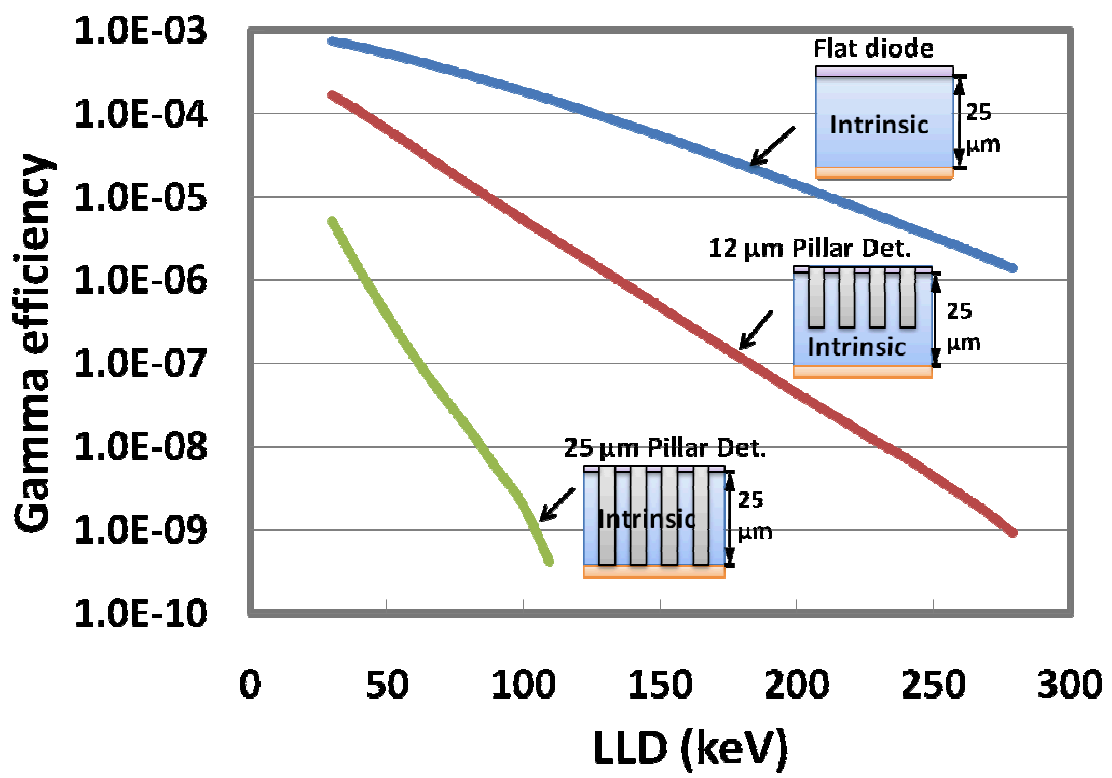

Figure 9 Gamma efficiencies of three devices as a function of low level discriminator 
Gamma discrimination is defined by the ratio of thermal neutron efficiency to gamma efficiency. Thermal neutron efficiency is calculated by dividing the total neutron counts collected from the detector by the incident neutron flux which was modeled using MCNP. By folding the results of MCNP simulations of the source, moderator, and laboratory, to account for room return, with the energy dependent ${ }^{10} \mathrm{~B}$ cross section, the incident equivalent thermal neutron flux was obtained. Figure 10 shows the neutron to gamma discrimination and neutron efficiency in relation with low level discriminator in the range of 80 to $110 \mathrm{keV}$. In this range, the thermal neutron efficiency is maintained at $21-22 \%$, while discrimination value ranges from $1.3 \times 10^{7}$ at $80 \mathrm{keV}$ to $5 \times 10^{8}$ at $110 \mathrm{keV}$. Besides pillar height, gamma sensitivity also depends on operation voltage, pillar sidewall roughness, fixed charges at pillar sidewall. Operation voltage determines the depletion portion of pillars which is responsible for the gamma absorption. Pillar sidewall roughness affects the amount of deposited energy through recombination rates of generated electron hole pairs. Fixed charges affect detector's capacitance which sets the noise floor in gamma and neutron measurements. These effects are currently under the investigation. ${ }^{6} \mathrm{LiF}$ based perforated semiconductor thermal neutron detectors under ${ }^{60} \mathrm{Co}$ radiation obtained gamma discrimination of $3 \times 10^{6}$ with LLD setting at $200 \mathrm{keV}$ and $1.6 \times 10^{7}$ with LLD setting at $500 \mathrm{keV}[14$, 15]. The signature gamma energy emitted from ${ }^{60} \mathrm{Co}$ is $1.3 \mathrm{MeV}$. For silicon, the linear attenuation coefficient is a function of incident gamma energy. Table 2 shows the relationship of incident gamma energies and corresponding amount of absorbed gamma-rays normalized to that of $1.3 \mathrm{MeV}$. The higher energy of incident gamma, the less amount of gamma absorbed. Given a certain silicon based detector, the absorbed gamma-rays of $60 \mathrm{keV}$ are 21 times higher than that of $1.3 \mathrm{MeV}$. LLD setting is crucial to determine neutron-to-gamma discrimination. For $25 \mu \mathrm{m}$ pillar detector, maximum LLD is set at $110 \mathrm{keV}$ as shown in Figure 10, because there are few gamma counts registered above that.

Table 2 Relationship of incident gamma energies and normalized gamma-rays absorbed by silicon

\begin{tabular}{|c|c|c|c|}
\hline Gamma source & $\begin{array}{c}\text { Gamma energy } \\
(\mathrm{keV})\end{array}$ & $\begin{array}{c}\text { Linear attenuation } \\
\text { coefficient }\left(\mathrm{cm}^{-1}\right)\end{array}$ & $\begin{array}{c}\text { Normalized amount of gamma- } \\
\text { rays absorbed by silicon }\end{array}$ \\
\hline${ }^{241} \mathrm{Am}$ & 60 & 0.68 & 21 \\
\hline${ }^{137} \mathrm{Cs}$ & 662 & 0.18 & 1.5 \\
\hline${ }^{60} \mathrm{Co}$ & 1300 & 0.14 & 1.0 \\
\hline
\end{tabular}

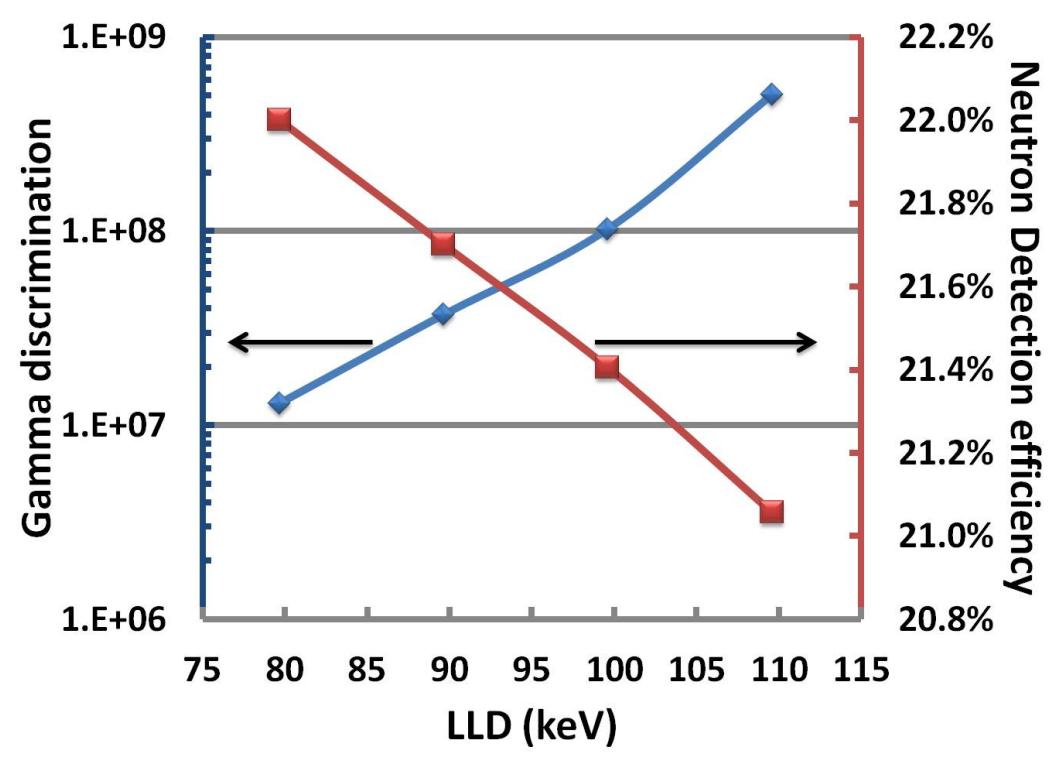

Figure 10 Gamma discrimination and thermal neutron detection efficiency versus low level discriminator for $25 \mu \mathrm{m}$ pillar detector 


\section{CONCLUSIONS}

MCNP and COMSOL simulation are conducted to optimize the design of pillar structured thermal neutron detectors in order to obtain high neutron-to-gamma discrimination while maintaining high thermal neutron detection efficiency. For a fixed pillar height, gamma discrimination is maximized by reducing the intrinsic layer thickness below the pillars. Higher doping density in the substrate makes gamma discrimination higher by reducing the diffusion length of minority carriers. For $25 \mu \mathrm{m}$ pillar detector, the gamma discrimination is measured as high as $1.3 \times 10^{7}$ with low level discriminator of $80 \mathrm{keV}$.

\section{ACKNOWLEDGMENT}

The authors would like to acknowledge Tim Graff and Cathy Reinhardt for their excellent support in the clean room fabrication. This work was performed under the auspices of the U.S. Department of Energy by Lawrence Livermore National Laboratory under Contract No. DE-AC52-07NA27344, LLNL-PROC-543492. This work has been supported by the US Department of Homeland Security, Domestic Nuclear Detection Office, under competitively awarded IAA HSHQDC-07-X-00213. This support does not constitute an express or implied endorsement on the part of the government.

\section{REFERENCES}

[1] G. Nelson, D. Reilly, "Gamma-Ray Interactions with Matter," Passive Nondestructive Analysis of Nuclear Materials, Los Alamos National Laboratory, NUREG/CR-5550, LAUR-90-732, pp. 27-42 (1991).

[2] G. F. Knoll, Radiation Detection and Measurement, Third ed. New York: Wiley, pp 518-520 (1999).

[3] L. Swiderski, M. Moszynski, D. Wolski, J. Iwanowska, T. Szczesniak, G. Pausch, C. Plettner, J. Stein, P. Schotanus, C. Hurlbut and J. Szabelski, "Comparison of neutron detection efficiency of a He-3 counter and a Boron-10 loaded liquid scintillator," IEEE Trans. on Nuclear Science, 57, pp. 2857-2861 (2010).

[4] A. M. Conway, R. J. Nikolic and T. F. Wang, "Numerical simulations of carrier transport in pillar structured solid state thermal neutron detector," International Semiconductor Device Research Conference, College Park, MD, December 12-14 (2007).

[5] R. J. Nikolic, A. M. Conway, C. E. Reinhardt, R. T. Graff, T. F. Wang, N. Deo and C. L. Cheung, "Pillar structured thermal neutron detectors," International Conference on Solid State and Integrated Circuit Technology (ICSICT), Beijing, China, October 20-23 (2008).

[6] R. J. Nikolic, C. L. Cheung, C. E. Reinhardt and T. F. Wang, "Roadmap for high efficiency solid-state neutron detectors," SPIE - International Symposium on Integrated Optoelectronic Devices, Vol. 6013, no. 1, pp. 36-44 (2005).

[7] A. M. Conway, T. F. Wang, N. Deo, C. L. Cheung and R. J. Nikolic, "Numerical simulations of pillar structured solid state thermal neutron detector: efficiency and gamma discrimination," IEEE Trans. on Nuclear Science, 56, pp. 2802-2807 (2009).

[8] R. J. Nikolic, A. M. Conway, C. E. Reinhardt, R. T. Graff, T. F. Wang, N. Deo and C. L. Cheung, "Pillar structured thermal neutron detector with 6:1 aspect ratio," Appl. Phys. Lett. 93, 133502 (2008).

[9] Q. Shao, A. M. Conway, L. F. Voss, D. P. Heineck, C. E. Reinhardt, R. T. Graff and R. J. Nikolic, "Leakage current quenching and lifetime enhancement in 3D pillar structured silicon PIN diodes," International Semiconductor Device Research Conference, College Park, MD, December 9-11 (2009).

[10]R. T. Kouzes, J. R. Ely, A. T. Lintereur, and D. L. Stephens, "Neutron detector gamma insensitivity criteria," Technical Report, PNNL-18903 (2009)

[11] “MCNP4C-Monte Carlo N-Particle Transport Code Systems,” Los Alamos National Laboratory (2000).

[12] http://www.comsol.com

[13] N. Deo, J. R. Brewer, C. E. Reinhardt, R. J. Nikolic and C. L. Cheung, "Conformal filling of silicon micro-pillar platform with 10boron,” J. Vac. Sci. Tech. B, vol. 26, no. 4, pp. 1309-1314 (2008).

[14] W. J. McNeil, S. L. Bellinger, T. C. Unruh, E. L. Patterson, J. K. Shultis, and D. S. McGregor, "Perforated diode fabrication for neutron detection," IEEE Nuclear Science Symposium Conference, pp. 3732-3735 (2006).

[15]D. S. McGregor, W. J. McNeil, S. L. Bellinger, T. C. Unruh and J. K. Shultis, "Microstructured semiconductor neutron detectors," Nuclear Instruments and Methods in Physics Research A 608, 125-131 (2009). 